RED GIANTS IN GLOBULAR CLUSTERS AND LARGE-SCALE VARIATIONS

IN THE GALAXY

B. E. J. Pagel

Royal Greenwich Observatory, Herstmonceux, Sussex, U.K.

Summary

This review concerns recent work on the determination of overall metallicities [ $\mathrm{Fe} / \mathrm{H}$ ] in a number of globular clusters and the systematics of mixing effects displayed (usually) by weak $\mathrm{CH}$ and strong $\mathrm{CN}$. Special attention is given to the globular cluster $\omega$ Centauri, where both metal abundance variations and mixing effects occur and are closely intertwined. Recent observations carried out at the Anglo-Australian Telescope by E.A. Mallia and D.C. Watts have revealed lange variations in the strength of metallic lines across the red giant branch of this cluster.

1. Large-scale Variations in the Galaxy

These have recently been discussed by Searle and Zinn (1978) and by Harris and Canterna (1979). The inner halo, within $10 \mathrm{kpc}$ of the Galactic centre, displays a wide range of metal deficiencies, $0.2<$ $[\mathrm{H} / \mathrm{Fe}]<2.3$, while the outer halo has a narrower range, 1.3 to 2.4 ; overall there is an abundance gradient similar to that in giant elliptical galaxies which has been given an interpretation by Larson (1976), and the distribution function is consistent with the simple enrichment model (Hartwick 1976). Whether these two statements apply to the outer halo is more controversial; more and better data are needed.

2. Methods of metallicity determination

There are two steps in this process: (i) the use of a ranking criterion, e.g. integrated spectral type(Arp 1965; van den Bergh 1967), Deutsch-Kinman class for individual giants (Kinman 1959), DDO colours

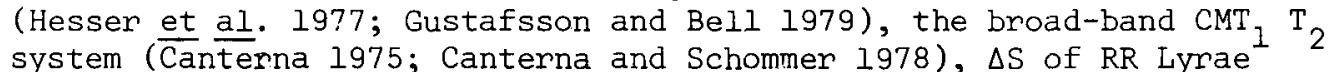
stars (Butler 1975), and the mean blanketing index <S> plotted against $M$ (Searle and Zinn 1978); the latter seems to be the most precise, with an error of \pm 0.1 dex. The second step (ii) is absolute cali-

817

Patrick A. Wayman (ed.), Highlights of Astronomy, Vol. 5, 817-826.

Copyright $\odot 1980$ by the IAU. 
Table 1

[ $\mathrm{H} / \mathrm{Fe}]$

\begin{tabular}{|c|c|c|c|c|c|c|c|}
\hline NGC & DDO & $\mathrm{CMT}_{1} \mathrm{~T}_{2}$ & $\Delta \mathrm{s}$ & $\langle S\rangle$ & Tio & $\begin{array}{c}\text { Gi spec } \\
\text { Lo } \\
\text { disp. }\end{array}$ & $\begin{array}{c}\text { Gi spec } \\
\text { Hi } \\
\text { disp. }\end{array}$ \\
\hline $\begin{array}{c}6341-\mathrm{M} 92 \\
\mathrm{~F} 2, \mathrm{C}\end{array}$ & $(2.2)$ & 2.2 & 2.2 & 2.0 & & & $\begin{array}{l}2.2,2.3 \\
2.4\end{array}$ \\
\hline $\begin{array}{c}7078-\mathrm{M} 15 \\
\text { F3, C }\end{array}$ & 1.9 & & 2.0 & 1.9 & & & 2.2 \\
\hline $\begin{array}{c}6656-M 22^{*} \\
\text { F5, C }\end{array}$ & \pm & 2.0 & 1.7 & & & 2.0 & 2.0 \\
\hline $\begin{array}{r}6397 \\
\text { F5, C }\end{array}$ & & & & & & & 2.0 \\
\hline
\end{tabular}

\begin{tabular}{|c|c|c|c|c|c|}
\hline $\begin{array}{r}6752 \\
\text { F6, B }\end{array}$ & & & & 1.7 & 1.7 \\
\hline $\begin{array}{c}5139-\omega \text { Cen* } \\
\text { F7, B }\end{array}$ & \pm & 1 to $2:$ & $\geqslant 0.6$ & $\begin{array}{l}1.0 \text { to } \\
1.5:\end{array}$ & $\begin{array}{l}1.0 \text { to } \\
1.7:\end{array}$ \\
\hline
\end{tabular}

$\begin{array}{llllll}5272-\mathrm{M} 3 \\ \mathrm{~F} 7, \mathrm{AB} & (1.5) & 1.4 & 1.6 & 1.7(\leqslant 1.0) & 1.8\end{array}$

\begin{tabular}{|c|c|c|c|c|c|c|c|}
\hline $\begin{array}{c}6205-\mathrm{Ml3} \\
\mathrm{F} 5, \mathrm{~A}\end{array}$ & 1.6 & & 1.0 & \multicolumn{3}{|c|}{$1.6(\leqslant 1.0)$} & $\begin{array}{l}1.4,1.5, \\
1.6\end{array}$ \\
\hline $\begin{array}{c}5904-M 5 * \\
\text { F6, A }\end{array}$ & 1.0 & & 1.0 & \multicolumn{3}{|l|}{1.2} & $1.4,1.5$ \\
\hline $\begin{array}{c}6171-\mathrm{M} 107 \\
\mathrm{Gl}, \mathrm{A}\end{array}$ & & & 0.8 & 0.6 & \multicolumn{3}{|l|}{0.9} \\
\hline $\begin{array}{l}\text { 104-47 Tuc* } \\
\text { G3, A }\end{array}$ & 0.4 & & & \multicolumn{2}{|c|}{$\leqslant 0.8$} & $0.8:$ & \multirow[t]{2}{*}{0.8} \\
\hline $\begin{array}{c}6838-M 71 \\
\text { G6, A }\end{array}$ & $(0.0)$ & 0.3 & 0.0 & 0.2 & 0.4 & & \\
\hline
\end{tabular}

*Var. CN 
bration, which can be carried out either by applying some of the above methods to field stars for which high-resolution curve-of-growth and model atmosphere studies are available, or by direct methods using model atmospheres and infra-red colours: Tio (Mould and McElroy 1978), suitable for more metal-rich clusters, and lowdispersion spectrum synthesis (Mallia 1977, 1978 and unpublished; Dickens and Bell 1976; Dickens, Bell and Gustafsson 1979). $\Delta S$ also appears to be quite a precise indicator of $[\mathrm{Fe} / \mathrm{H}]$, despite the fact that it refers to $\mathrm{Ca}$ and not to $\mathrm{Fe}$ (Butler and Deming 1979), but there could be a few reservations related to correction for interstellar $\mathrm{K}$ and also the Blazhko effect (Romanov 1979)。

Table 1 gives results for selected clusters derived by the various methods. Sources for high-resolution results are:- M92: Pagel (1966) from spectra by Helfer et al. (1959); Cohen (1979). M15: Cohen (1979). M22, 6397, 6752, $\bar{\omega}$ Cen: Mallia (1977, 1978, 1979). M3: Cohen (1978). M13: Cohen (1978), Wallerstein et al. (1979a), Griffin (1979). 47. Tuc: Mallia (1979). Apart from odd results for $M I 3(\Delta S)$ and 47 Tuc (DDO), the agreement is generally good. Note, however, the enormous range in $[\mathrm{Fe} / \mathrm{H}]$ embraced by Deutsch-Kinman Class A. 


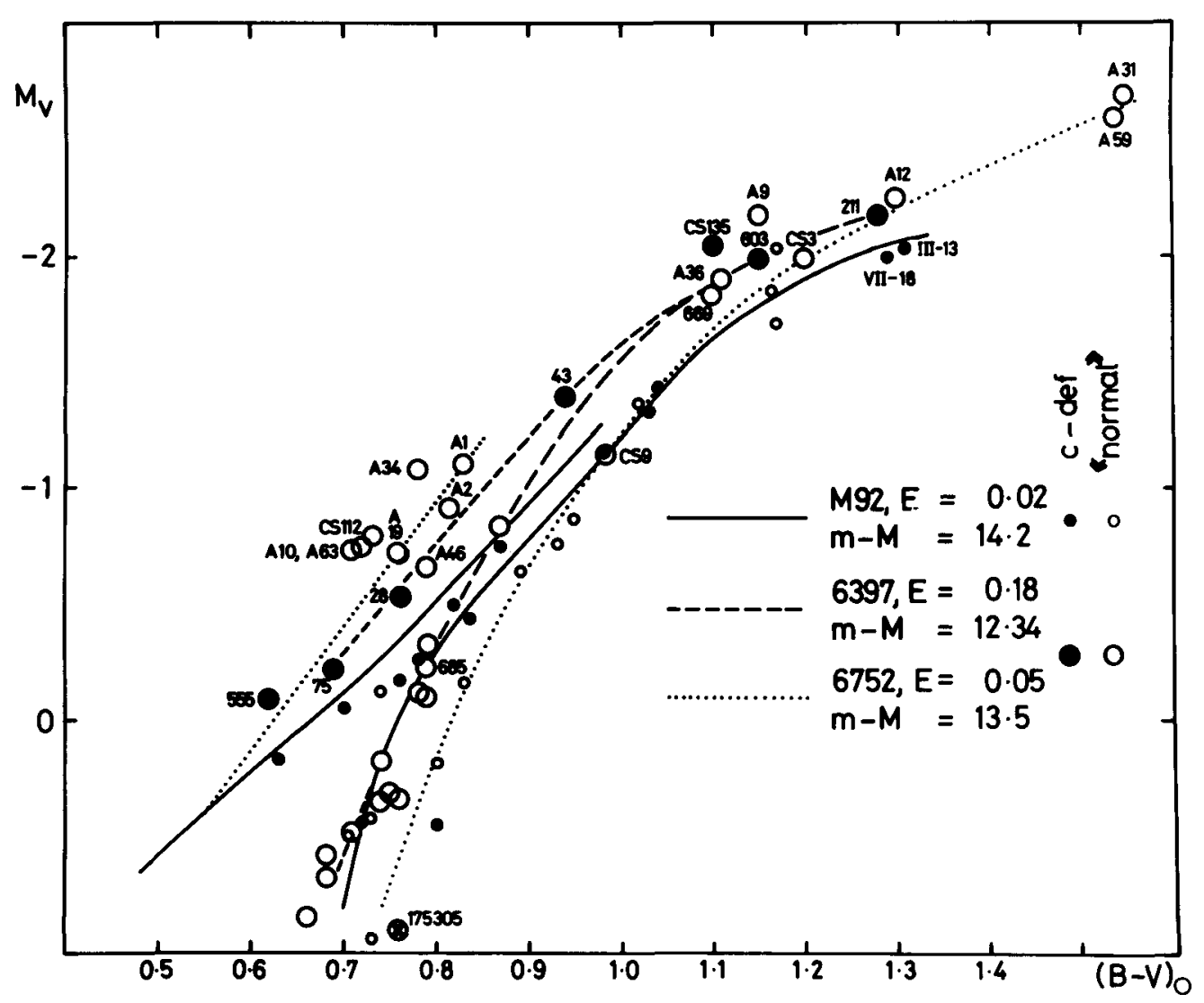

Fig. I Incidence of weak G-band effect or carbon deficiency in the HR diagrams of M92, NGC 6397 and NGC 6752. (Numbers preceded by 'A' or 'CS' refer to 6752 , others to 6397. ) The field star HD 175305, with normal C/Fe (Wallerstein et al. 1979b), is also shown; from its position it appears to be a subgiant rather than a red horizontal branch star. 


\section{Mixing Effects}

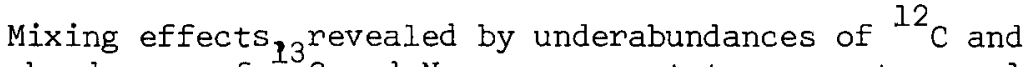
over-abundances of ${ }^{13} \mathrm{C}$ and $\mathrm{N}$, are present to a greater or lesser extent in nearly all giants, but extreme cases are more common in Population II objects. Many of the effects observed (apart from the comparatively rare $\mathrm{CH}$ stars) receive a promising explanation from the theory of Sweigart and Mengel (1979) who suggest mixing by meridional circulation due to internal rotation, which becomes effective when $M<-0.5$ or so and implies that carbon deficiency and nitrogen over-abundance should occur in all stars that either are or have been on the upper part of the red giant branch (RGB). This prediction can be compared with the "weak $G$ band" effect observed in the more metal-deficient clusters by Norris and Zinn (1977), Mallia (1977, 1978) and Bell et al. (1979). Fig. I shows the incidence of weak $G$ bands or carbon deficiency in three typical clusters: in NGC 6397, weak $G$ bands occur entirely on the asymptotic giant branch (AGB) and in M92 they show a preference for the AGB, whereas in 6752 only one star on the upper giant branch shows a weak $G$ band. At the tip of the giant branch, the effect is not directly visible because the stars are too cool; here spectrum synthesis has to be used and the resulting $[\mathrm{C} / \mathrm{Fe}]$ depends on the temperature scale (Bell et al. 1979). Probably M92 and 6397 behave more or less as expected from meridional mixing, with $[\mathrm{C} / \mathrm{Fe}] \simeq-1$ in the brighter giants, but the situation in 6752 is more doubtful. 
In more metal-rich clusters, the same effect chiefly manifests itself in strong CN, e.g. 47 Tuc (Dickens et al. 1979; Mallia 1978, 1979), where stars with and without readily detectable violet $C N$ bands are found together all along the RGB for $\mathrm{M}<+0.5$ or so (Fig. 2). A unique solution for the $C, N, O$ abundancés is difficult to obtain for such strong-lined stars; the solution preferred by Dickens et al. is that carbon is normal except on the AGB, with nitrogen varying from underabundant $([\mathrm{N} / \mathrm{Fe}]<0)$ to overabundant by a factor 5 . However, further complications are introduced by uncertainty in the oxygen abundance (Mallia 1979).

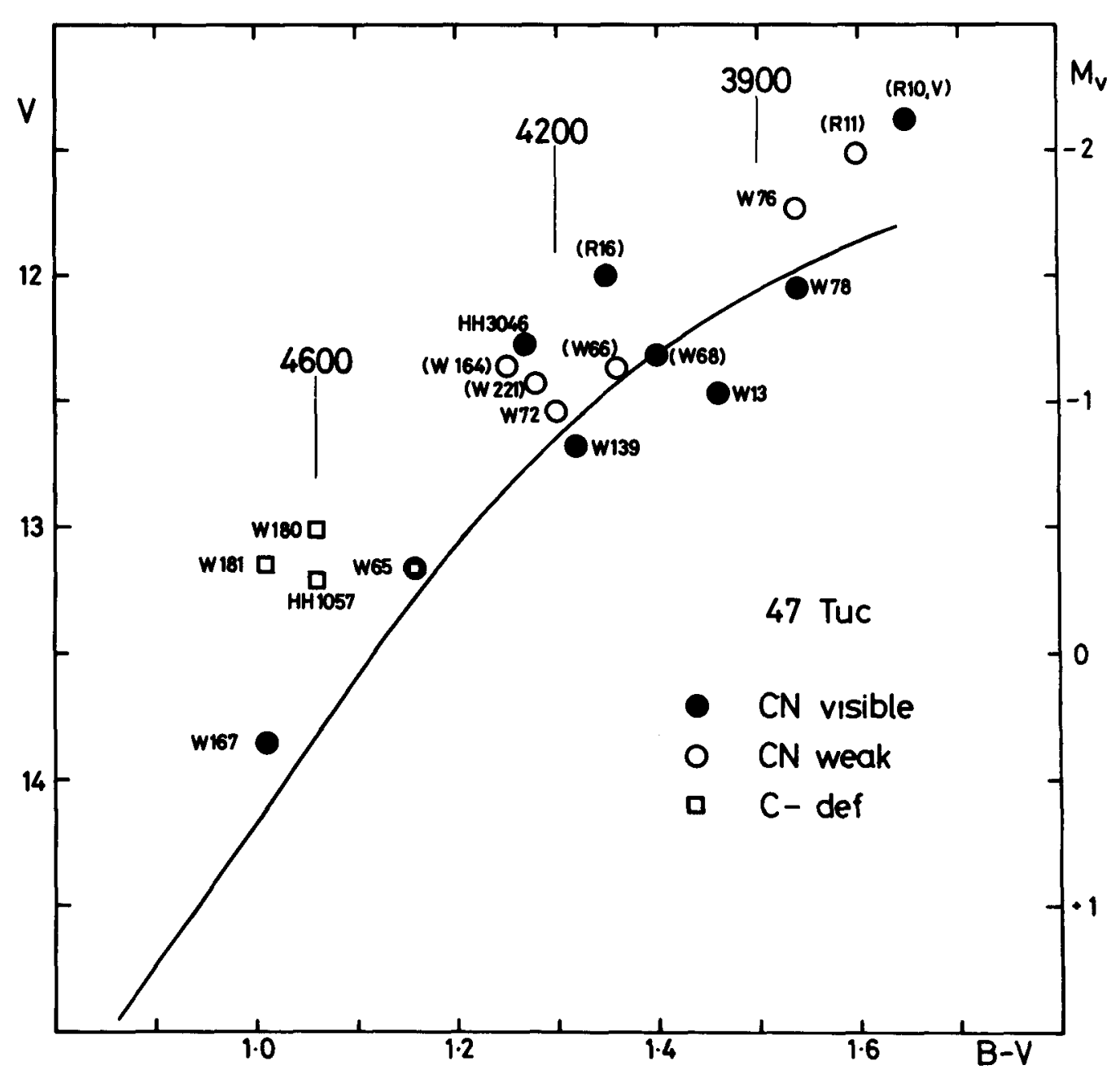

Fig. 2 HR diagram of 47 Tuc showing incidence of mixing effects. Effective temperatures are from Dickens et al. (1979). Stars noted in brackets have only poor colour determinations. 


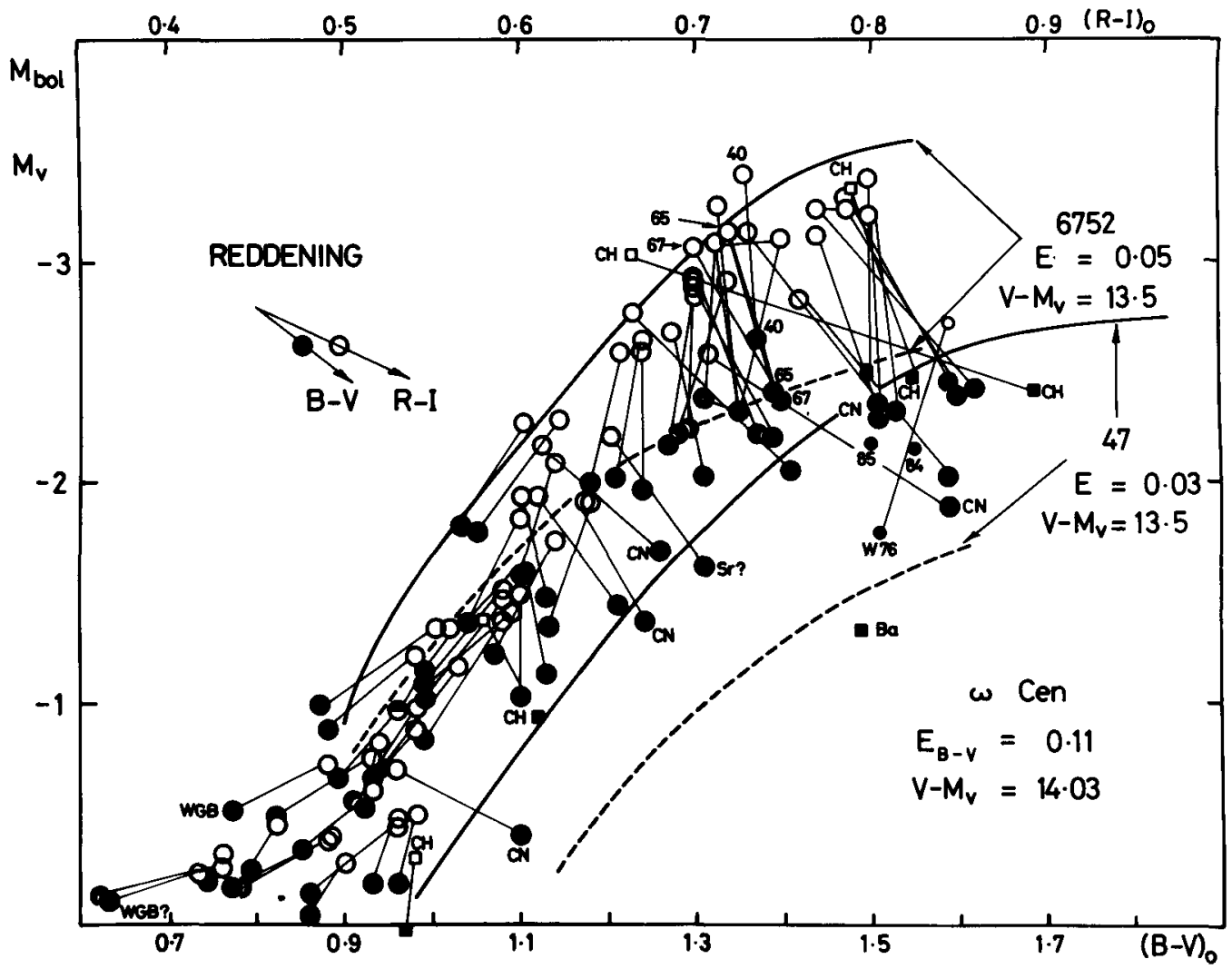

Fig. 3 Composite HR diagram for $\omega$ Cen (see text). W76, in 47 Tuc, is shown for comparison.

\section{4. $\omega$ Centauri}

Fig. 3 shows a rather complicated $\mathrm{HR}$ diagram in both $\mathrm{M}_{\mathrm{v}}, \mathrm{B}-\mathrm{V}$ (filled circles, broken lines) and $M_{\text {bol, }}, \mathrm{R}-\mathrm{I}$ (open circles, solid lines); the lines refer to two comparison clusters, NGC 6752 and 47 Tuc, after Eggen (1972). R-I has been converted to the Johnson scale. Some of the mixing anomalies discussed by Norris and Bessell (1975, 1977, 1978), Dickens and Bell (1976) and Bessell and Norris (1976) are indicated. The diagram shows the well-known spread in $B-V$, with strong $C N$ stars on the red side, and a rather small spread in R-I, which has led Bessell and Norris to suggest that the major 


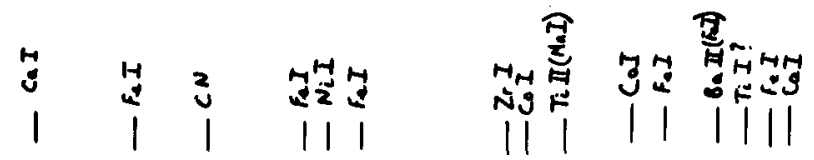

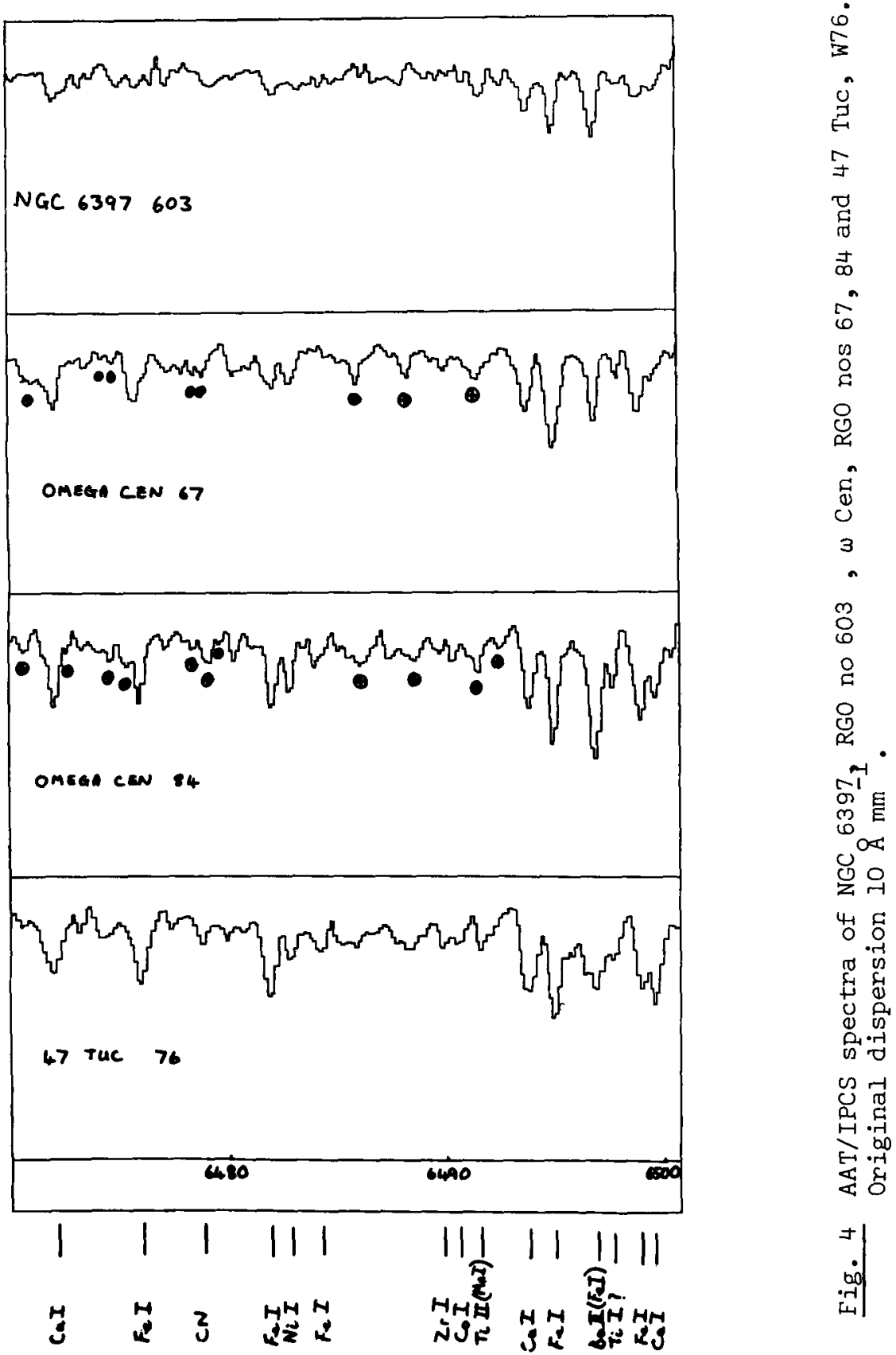


cause of the spread in $\mathrm{B}-\mathrm{V}$ is the mixing effect. On the other hand, studies of RR Lyrae stars by Freeman and Rodgers (1975) and Butler et al (1978) imply a large range in underlying metal abundance, roughly $1<[\mathrm{H} / \mathrm{Fe}]<2$. The question therefore arises of what range in metal abundance exists across the red giant branch.

A preliminary answer to this question can be given on the basis of $10 \mathrm{~A} / \mathrm{mm}$ spectra of the "blue" stars RGO 40,65, 67 and the "red" stars RGO 85, 84 taken earlier this year by E. A. Mallia and D. C. Watts with the Anglo-Australian Telescope and the UCL Image Photon Counting System (Fig. 4). These show very marked differences in metallic line strength, with RGO 84 (a star without marked CN enhancement; see Dickens and Bell 1976) coming very close to the star W 76 in 47 Tuc. A preliminary model-atmosphere abundance analysis by Mallia gives $[\mathrm{Fe} / \mathrm{H}]=-1.7$ for the "blue" stars and -1.0 for the "red" stars (assuming $T=04000 \mathrm{~K}, \log g=0.75$ ). Thus a substantial range in metal abundance occurs across the red giant branch, although the preliminary $[\mathrm{Fe} / \mathrm{H}]$ values neither cover the complete range deduced from RR Lyrae stars nor offer any straightforward explanation for the apparent narrowness of the giant branch in $R-I . *$

I thank the SRC Panel for the Allocation of Telescope Time for the award of time on the AAT for this programme and E. A. Mallia for providing the bulk of the new results reported here.

*After presentation of this paper, a recent investigation of the red giant branch by Rodgers et al.(1979), using Searle and Zinn's $\langle s\rangle$ parameter, came to my attention. Rodgers et al. derive $-1.8 \leqslant$ $[\mathrm{Fe} / \mathrm{H}] \leqslant-0.8$, which is not too different from the results reported here. 


\section{REFERENCES}

Arp, H. C. 1965, in Galactic Structure, ed. A. Blaauw and

M. Schmidt, Univ. of Chicago Press, p. 410.

Bell, R. A., Dickens, R. J., and Gustafsson, B. 1979, Astrophys. J., 229,604 .

Bessell, M. S., and Norris, J. 1976, Astrophys. J., 208, 369.

Butler, D. D. 1975, Astrophys. J., 200, 68 .

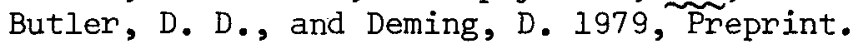

Butler, D. D., Dickens, R. J., and Epps, E. A., 1978, Astrophys. J., $225,148$.

Canterna, R. 1975, Astrophys. J. Let., 200, L63.

Canterna, R., and Schommer, R. A. 1978, Astrophys. J., Let. 219, L119.

Cohen, J。G. 1978, Astrophys. J., 223, 487.

Cohen, J. G. 1979, Astrophys. J., 231,751 .

Dickens, R. J., and Bell, R. A. 1976, Astrophys. J., 207, 506.

Dickens, R. J., Bell, R. A., and Gustafsson, B. 1979, Astrophys. J., 232,428 .

Eggen, 0. J. 1972, Astrophys. J., 172, 639.

Freeman, K. C., and Rodgers, A. W. 1975, Astrophys. J. Let., 101, L7l. Griffin, R. 1979, Mon. Not. R. astr. Soc., 187, 269.

Gustafsson, B., and Bell, R. A. 1979, Astron. Astrophys., 74, 313.

Harris, W. E., and Canterna, R. 1979, Astrophys. J. Let. 2每, Ll9.

Hartwick, F. D. A. 1976, Astrophys. J., 209, 418.

Helfer, H. L., Wallerstein, G., and Greenstein, J. L. 1959,

Astrophys. J., 129, 700 .

Hesser, J. E., Hartwick, F. D. A., and McClure, R. D. 1977,

Astrophys, J. Sup., 33, 471 .

Kinman, T. D. 1959, Mon. Not. R. astr. Soc., 119, 538.

Larson, R. 1976, Mon. Not. R. astr. Soc., 176, 31

Mallia, E. A. 1977, Astron. Astrophys., 60, 195.

Mallia, E. A. 1978, Astron. Astrophys.. 70. 115

Mallia, E. A. 1979, private communication

Mould. J. R., and McElroy, D. B. 1978, Astrophys. J., 221, 580.

Norris, J., and Bessell, M. S. 1975, Astrophys. J. Let., 201, L75.

Norris, J., and Bessell, M. S. 1976, Astrophys. J. Let., 2 2II, L91.

Norris, J., and Bessell, M. S. 1978, Astrophys. J. Let., 225, L49. Norris, J., and Zinn, R. 1977, Astrophys. J., 162, 841.

Pagel, B. E. J. 1966, in Colloquium on Late-Type Stars, ed. M. Hack, Trieste, P.133.

Rodgers, A. W., Freeman, K. C., Harding, P., and Smith, G. H. 1979, Astrophys. J., 232, 169.

Romanov, Y. S. 197 $\widetilde{9}$, private communication.

Searle, L., and Zinn, R. 1978, Astrophys. J., 225, 357.

Sweigart, A. V., and Mengel, J. G. 1979, Astrophys. J., 229, 624. van den Bergh, S., 1967, Astron. J., 72, 70.

Wallerstein, G., Pilachowski, C. A., and Leep, E. M. 1979a, in

Les Elements et leurs Isotopes dans 1'Univers, 22nd Astrophysical

Colloquium, Liege, ed. N. Grevesse.

Wallerstein, G., Pilachowski, C., Gerend, D., Scott, B., and Canterna,

R. 1979b, Mon. Not. R. Astr. Soc., 186, 691 . 